\title{
THE INITIAL TRACE OF A SOLUTION OF THE POROUS MEDIUM EQUATION ${ }^{1}$
}

BY

\author{
D. G. ARONSON AND L. A. CAFFARELLI
}

\begin{abstract}
Let $u=u(x, t)$ be a continuous weak solution of the porous medium equation in $\mathbf{R}^{d} \times(0, T]$ for some $T>0$. We show that corresponding to $u$ there is a unique nonnegative Borel measure $\rho$ on $\mathbf{R}^{d}$ which is the initial trace of $u$. Moreover, we show that the initial trace $\rho$ must belong to a certain growth class. Roughly speaking, this growth restriction shows that there are no solutions of the porous medium equation whose pressure grows, on average, more rapidly then $|x|^{2}$ as $|x| \rightarrow \infty$.
\end{abstract}

Introduction. Let $u=u(x, t)$ be a nonnegative solution of the equation of heat conduction

$$
\partial u / \partial t=\Delta u
$$

in $S_{T} \equiv \mathbf{R}^{d} \times(0, T]$ for some $T>0$. A consequence of the Widder representation theorem is the existence of a unique nonnegative Borel measure $\rho$ such that

$$
\lim _{t \downarrow 0} \int_{\mathbf{R}^{d}} u(x, t) \varphi(x) d x=\int_{\mathbf{R}^{d}} \varphi(x) \rho(d x)
$$

for all test functions $\varphi \in C_{0}\left(\mathbf{R}^{d}\right)$ [1]. That is, every nonnegative solution $u$ of (1) has a unique Borel measure $\rho$ as initial trace in the sense of (2). The measure $\rho$ is $\sigma$-finite and satisfies the growth condition

$$
\int_{\mathbf{R}^{d}} e^{-\mid \xi \xi^{2} / 4 T} \rho(d \xi)<+\infty .
$$

In this paper we establish the existence of a unique initial trace $\rho$ for each continuous nonnegative weak solution of the porous medium equation

$$
\partial u / \partial t=\Delta\left(u^{m}\right)
$$

in $S_{T}$. Here $m>1$ is a constant. In addition, we show that the initial trace $\rho$ must satisfy a growth condition analogous to (3) which limits the amount of mass which $\rho$ can place at $|x|=+\infty$. Specifically, there exists a constant $C>0$ depending only on $d$ and $m$ such that

$$
\int_{\{x:|x|<r\}} \rho(d x) \leqslant C\left\{r^{\kappa /(m-1)} T^{-1 /(m-1)}+T^{d / 2} u^{\kappa / 2}(0, T)\right\}
$$

Received by the editors October 25, 1982.

1980 Mathematics Subject Classification. Primary 35K55, 76S05; Secondary 35K15.

'This work was supported, in part, by National Science Foundation Grants MCS 80-02392 and MCS 79-00985. 
where

$$
\kappa=d(m-1)+2 .
$$

Roughly speaking, (5) means that, on average, $u(x, 0)$ cannot grow faster than $|x|^{2 /(m-1)}$ as $|x| \rightarrow \infty$.

If $u=u(x, t)$ is a solution of (4) with $u(x, 0) \in L^{1}\left(\mathbf{R}^{d}\right)$ and if $u$ decays sufficiently rapidly as $|x| \rightarrow \infty$, then it is easy to show that $\|u(\cdot, t)\|_{L^{\prime}\left(\mathbf{R}^{d}\right)}$ is constant, that is, mass is conserved. Here we are concerned with solutions whose initial traces are not necessarily integrable and which may even grow as $|x| \rightarrow \infty$. In this context the conservation of mass is replaced by a Harnack-type inequality which gives a sharp estimate for the mass in a ball of radius $r$ at time $t$ in terms of the density at the center of the ball at a later time (Theorem 3.1). From this result we deduce our main results on existence and growth of the initial trace (Theorem 4.1). An additional argument is needed to establish the uniqueness of the trace and this is given in $\S 4$. $\S \S 1$ and 2 are devoted to preliminary matters. In $\S 1$ we derive some comparison principles and in $\$ 2$ we show that solutions of (4) whose initial values have compact support behave almost radially. In [8] Maura Ughi extends our results to equations of the form $\partial u / \partial t=\Delta \varphi(u)$ under suitable hypotheses on $\varphi$.

Let $\rho$ be a nonnegative Borel measure which satisfies (3) for some $T>0$ and let

$$
g(x, t)=(4 \pi t)^{-d / 2} e^{-|x|^{2} / 4 t} .
$$

It is known [1] that the function

$$
u(x, t) \equiv \int_{\mathbf{R}^{d}} g(x-\xi, t) \rho(d \xi)
$$

is a nonnegative solution of (1) in $S_{T}$ whose initial trace is $\rho$. Recently Bénilan, Crandall and Pierre [4] have constructed solutions of the porous medium equation (4) whose initial traces are measures which satisfy (5). This extends some earlier work by Kalashnikov [7] for measures with smooth densities on $\mathbf{R}$. Moreover, it shows that the condition (5) is not only necessary but also sufficient for existence of solutions of (4). Even more recently Dahlberg and Kenig [9] have proved uniqueness for the solutions constructed in [4].

1. Comparison principles. Throughout this paper we will be dealing with continuous weak solutions of the porous medium equation

$$
\partial u / \partial t=\Delta\left(u^{m}\right)
$$

in $S_{T} \equiv \mathbf{R}^{d} \times(0, T]$ for some $T>0$. We always assume that $m>1$. Specifically, a function $u=u(x, t)$ is said to be a continuous weak solution of (1.1) in $S_{T}$ if $u$ is nonnegative and continuous in $S_{T}$ and satisfies the integral identity

$$
\iint_{\mathbf{R}^{d} \times\left(\tau_{1}, \tau_{2}\right)}\left(u^{m} \Delta \varphi+u \frac{\partial \varphi}{\partial t}\right) d x d t=\left.\int_{\mathbf{R}^{d}} u \varphi\right|_{t=\tau_{2}} d x-\left.\int_{\mathbf{R}^{d}} u \varphi\right|_{t=\tau_{1}} d x
$$

for all $\tau_{i}$ such that $0<\tau_{1}<\tau_{2} \leqslant T$ and all $\varphi \in C^{2,1}\left(S_{T}\right)$ such that $\varphi(\cdot, t)$ has compact support for all $t \in\left[\tau_{1}, \tau_{2}\right]$. 
Among the main tools which we employ in this work are various comparison principles for weak solutions of (1.1). Usually such comparison principles are obtained as byproducts of the existence and uniqueness theory. However, sometimes one can obtain a comparison principle directly. This can be done, for example, in the case of weak solutions of certain boundary value problems. We formulate the appropriate notions below and then show that continuous weak solutions of (1.1) in $S_{T}$ can also be interpreted as weak solutions of boundary value problems.

For arbitrary $\xi \in \mathbf{R}^{d}, r \in \mathbf{R}^{+}$, and $\tau_{i}$ such that $0<\tau_{1}<\tau_{2} \leqslant T$, let

$$
\Gamma=B_{r}(\xi) \times\left(\tau_{1}, \tau_{2}\right],
$$

where $B_{r}(\xi)=\left\{x \in \mathbf{R}^{d}:|x-\xi|<r\right\}$. The parabolic boundary, $\partial_{p} \Gamma$, of $\Gamma$ is defined by

$$
\partial_{p} \Gamma \equiv \vec{\Gamma} \backslash \Gamma
$$

Let $g \in C\left(\partial_{p} \Gamma\right)$ be a given nonnegative function and consider the boundary value problem

$$
\partial v / \partial t=\Delta\left(v^{m}\right) \quad \text { in } \Gamma, \quad v=g \text { on } \partial_{p} \Gamma .
$$

A function $v=v(x, t)$ is said to be a weak solution of problem (1.4) in $\Gamma$ if $v \in C\left(\left[\tau_{1}, \tau_{2}\right]: L^{1}\left(B_{r}\right)\right) \cap L^{\infty}(\Gamma), v \geqslant 0$, and $v$ satisfies the integral identity

$$
\begin{aligned}
\iint_{\Gamma}\left(v^{m} \Delta \varphi+v \frac{\partial \varphi}{\partial t}\right) & d x d t \\
& =\int_{\tau_{1}}^{\tau_{2}} \int_{\partial B_{r}} g^{m} \frac{\partial \varphi}{\partial \nu} d s d t+\left.\int_{B_{n}} v \varphi\right|_{t=\tau_{2}} d x-\left.\int_{B_{n}} g \varphi\right|_{t=\tau_{1}} d x
\end{aligned}
$$

for all $\varphi \in C^{1,0}(\bar{\Gamma}) \cap C^{2,1}(\Gamma)$ with $\varphi=0$ on $\partial B_{r} \times\left[\tau_{1}, \tau_{2}\right]$. In $(1.5), \partial / \partial \nu$ denotes the exterior normal derivative on $\partial B_{r}$.

There is an a priori comparison principle for solutions of (1.4). This is the content of the next result, which is simply a restatement of Theorem 12 of [3].

Proposition 1.1 (Local Comparison Principle). For $i=1$ and 2 , let $v_{i}$ denote $a$ solution of (1.4) with boundary values $g_{i}$. If $g_{1} \leqslant g_{2}$ on $\partial_{p} \Gamma$, then $v_{1} \leqslant v_{2}$ in $\bar{\Gamma}$.

Proposition 1.1 is proved in [3] for $x \in \mathbf{R}$, but there is no difficulty in extending the proof for $x \in \mathbf{R}^{d}$. A proof for $x \in \mathbf{R}^{d}$ is also given in [9]. The local comparison principle, Proposition 1.1, can be applied to continuous weak solutions of (1.1) since, as we show next, every such solution is also a solution of (1.4) with boundary values $g=u \mid \partial_{p} \Gamma$.

Proposition 1.2. Let $u$ be a continuous weak solution of $(1.1)$ is $S_{T}$ for some $T>0$. For any cylinder $\Gamma$ of the form (1.3), $u$ is a weak solution of (1.4) in $\Gamma$ with boundary values $g=u \mid \partial_{p} \Gamma$.

Proof. It suffices to show that the weak solutions of (1.1) satisfy (1.5). Fix $\xi \in \mathbf{R}^{d}$, $r \in \mathbf{R}^{+}, 0<\tau_{1}<\tau_{2} \leqslant T$, and let $\varphi$ be a $C^{1,0}(\bar{\Gamma}) \cap C^{2,1}(\Gamma)$ function with $\varphi=0$ on 
$\partial B_{r} \times\left[\tau_{1}, \tau_{2}\right]$. For each $\varepsilon \in(0, r)$ and $\eta \in[0, \varepsilon)$ let $\psi_{\varepsilon \eta}$ denote the $C\left(\mathbf{R}^{d}\right)$ function which satisfies $\psi_{\varepsilon \eta}(x)=\Psi_{\varepsilon \eta}(|x|)$ with

$$
\Psi_{\varepsilon \eta}(\rho)= \begin{cases}1 & \text { for } 0 \leqslant \rho \leqslant r-\varepsilon \\ 0 & \text { for } \rho \geqslant r-\eta\end{cases}
$$

and

$$
\Delta \psi_{\varepsilon \eta}=|x|^{-d+1}\left(|x|^{d-1} \Psi_{\varepsilon \eta}^{\prime}\right)^{\prime}=0 \text { in } B_{r-\eta} \backslash \bar{B}_{r-\varepsilon} .
$$

One can compute $\nabla \psi_{\varepsilon \eta}$ explicitly, and it turns out that

$$
\nabla \psi_{\varepsilon \eta}(x)= \begin{cases}-W_{\varepsilon \eta}|x|^{-d} x & \text { for } x \in B_{r-\eta} \backslash \bar{B}_{r-\varepsilon}, \\ 0 & \text { for } x \in B_{r-\varepsilon} \cup\left(\mathbf{R}^{d} \backslash \bar{B}_{r-\eta}\right),\end{cases}
$$

where, for $d>2$,

$$
W_{\varepsilon \eta}=\frac{(d-2)(r-\eta)^{d-2}(r-\varepsilon)^{d-2}}{(r-\eta)^{d-2}-(r-\varepsilon)^{d-2}} .
$$

As a distribution on $\mathbf{R}^{d}, \Delta \psi_{\varepsilon \eta}$ is a signed measure. Specifically,

$$
\left(\Delta \psi_{\varepsilon \eta}\right)(d x)=W_{\varepsilon \eta}\left\{\delta_{r-\eta}(d|x|)-\delta_{r-\varepsilon}(d|x|)\right\} d \omega,
$$

where $d \omega$ denotes the surface element on the unit ball in $\mathbf{R}^{d}$ and $\delta_{\lambda}(d|x|)$ denotes the Dirac measure concentrated at $|x|=\lambda$. Similar expressions can be derived for $d=1$ and 2. Let $k_{\gamma}(x)$ be a $C^{\infty}$ symmetric averaging kernel with support in $B_{\gamma}(0)$ and define

$$
\psi_{\varepsilon \eta}^{\gamma}(x)=\int_{\mathbf{R}^{d}} k_{\gamma}(x-\xi) \psi_{\varepsilon \eta}(\xi) d \xi
$$

Then

$$
\Delta \psi_{\varepsilon \eta}^{\gamma}(x)=\int_{\mathbf{R}^{d}} k_{\gamma}(x-\xi)\left(\Delta \psi_{\varepsilon \eta}\right)(d \xi)
$$

In particular, if $\gamma$ is sufficiently small then $\Delta \psi_{\varepsilon \eta}^{\gamma}$ is supported by two disjoint annular regions: one containing $\partial B_{r-\varepsilon}$ and the other containing $\partial B_{r-\eta}$. The function $\varphi \psi_{f \eta}^{\gamma}$ is an admissible test function in (1.2). Therefore, we have

$$
\iint_{\mathbf{R}^{d} \times\left(\tau_{1}, \tau_{2}\right)}\left\{u^{m} \Delta\left(\varphi \psi_{\varepsilon \eta}^{\gamma}\right)+u \psi_{\varepsilon \eta}^{\gamma} \frac{\partial \varphi}{\partial t}\right\} d x d t=\left.\int_{\mathbf{R}^{d}} u \varphi \psi_{\varepsilon \eta}^{\gamma}\right|_{t=\tau_{2}} d x-\left.\int_{\mathbf{R}^{d}} u \varphi \psi_{\varepsilon \eta}^{\gamma}\right|_{t=\tau_{1}} d x .
$$

Since $u^{m}$ is continuous we can let $\gamma \downarrow 0$ in (1.6) to obtain

$$
\begin{gathered}
\iint_{\Gamma}\left(u^{m} \psi_{\varepsilon \eta} \Delta \varphi+\right. \\
\left.+u \psi_{\varepsilon \eta} \frac{\partial \varphi}{\partial t}\right) d x d t+2 \int_{\tau_{1}}^{\tau_{2}} d t \int_{B_{r-\eta} \backslash \bar{B}_{r-\varepsilon}} u^{m} \nabla \psi_{\varepsilon \eta} \cdot \nabla \varphi d x \\
+W_{\varepsilon \eta} \int_{\tau_{1}}^{\tau_{2}} d t\left\{\int_{\partial B_{r-\eta}} u^{m} \varphi d \omega-\int_{\partial B_{r-\varepsilon}} u^{m} \varphi d \omega\right\} \\
=\left.\int_{B_{r}} u \varphi \psi_{\varepsilon \eta}\right|_{t=\tau_{2}} d x-\left.\int_{B_{r}} u \varphi \psi_{\varepsilon \eta}\right|_{t=\tau_{1}} d x .
\end{gathered}
$$


Now let $\eta \downarrow 0$. Because $\varphi=0$ on $\partial B_{r} \times\left[\tau_{1}, \tau_{2}\right]$ it follows that

$$
\begin{aligned}
\iint_{\Gamma}\left(u^{m} \psi_{\varepsilon 0} \Delta \varphi+u \psi_{\varepsilon 0}\right. & \left.\frac{\partial \varphi}{\partial t}\right) d x d t+I_{\varepsilon}+J_{\varepsilon} \\
& =\left.\int_{B_{r}} u \varphi \psi_{\varepsilon 0}\right|_{t=\tau_{2}} d x-\left.\int_{B_{r}} u \varphi \psi_{\varepsilon 0}\right|_{t=\tau_{1}} d x,
\end{aligned}
$$

where

$$
I_{\varepsilon} \equiv 2 \int_{\tau_{1}}^{\tau_{2}} d t \int_{B_{r} \backslash \bar{B}_{r-\varepsilon}} u^{m} \nabla \psi_{\varepsilon 0} \cdot \nabla \varphi d x
$$

and

$$
J_{\varepsilon} \equiv-W_{\varepsilon 0} \int_{\tau_{1}}^{\tau_{2}} d t \int_{\partial B_{r-\varepsilon}} u^{m} \varphi d \omega
$$

If we introduce spherical coordinates on $\mathbf{R}^{d}$ then

$$
I_{\varepsilon}=-2 W_{\varepsilon 0} \int_{\tau_{1}}^{\tau_{2}} d t \int_{r-\varepsilon}^{r}\left(\left.\int_{\omega} u^{m} \frac{\partial \varphi}{\partial \nu}\right|_{|x|=\rho} d \omega\right) d \rho .
$$

Observe that

$$
\lim _{\varepsilon \downarrow 0} \varepsilon W_{\varepsilon 0}=r^{d-1}
$$

Therefore

$$
\lim _{\varepsilon \downarrow 0} I_{\varepsilon}=-\left.2 r^{d-1} \int_{\tau_{1}}^{\tau_{2}} d t \int_{\omega} u^{m} \frac{\partial \varphi}{\partial \nu}\right|_{|x|=r} d \omega .
$$

On the other hand, since $\varphi=0$ for $|x|=r$ we can write

$$
J_{\varepsilon}=\left.W_{\varepsilon 0} \int_{\tau_{1}}^{\tau_{2}} d t \int_{\omega} u^{m}\right|_{|x|=r-\varepsilon}\left\{\left.\varphi\right|_{|x|=r}-\left.\varphi\right|_{|x|=r-\varepsilon}\right\} d \omega .
$$

In view of (1.8) and the continuity of $u^{m}$ it follows that

$$
\lim _{\varepsilon \downarrow 0} J_{\varepsilon}=\left.r^{d-1} \int_{\tau_{1}}^{\tau_{2}} d t \int_{\omega} u^{m} \frac{\partial \varphi}{\partial \nu}\right|_{|x|=r} d \omega .
$$

Thus if we let $\varepsilon \downarrow 0$ in (1.7) we obtain

$$
\begin{aligned}
\iint_{\Gamma}\left(u^{m} \Delta \varphi+u \frac{\partial \varphi}{\partial t}\right) & d x d t-\left.\int_{\tau_{1}}^{\tau_{2}} d t \int_{\omega} u^{m} \frac{\partial \varphi}{\partial \nu}\right|_{|x|=r} r^{d-1} d \omega \\
& =\left.\int_{B_{r}} u \varphi\right|_{t=\tau_{2}} d x-\left.\int_{B_{r}} u \varphi\right|_{t=\tau_{1}} d x,
\end{aligned}
$$

which is equivalent to (1.5) with $g=u \mid \partial_{p} \Gamma$.

Let $u$ be a continuous weak solution of (1.1) in $S_{T}$ and let $\zeta$ be a fixed $C_{0}^{\infty}\left(\mathbf{R}^{d}\right)$ function which takes its values in $[0,1]$. Fix $\tau \in(0, T)$ and consider the initial value problem

$$
\partial w / \partial t=\Delta\left(w^{m}\right) \quad \text { in } \mathbf{R}^{d} \times(\tau, T], \quad w(\cdot, \tau)=\zeta u(\cdot, \tau) \quad \text { in } \mathbf{R}^{d} .
$$


A weak solution of (1.9) is a nonnegative function $w=w(x, t)$ which satisfies the integral identity

$$
\iint_{\mathbf{R}^{d} \times(\tau, T)}\left(w^{m} \Delta \varphi+w \frac{\partial \varphi}{\partial t}\right) d x d t=\left.\int_{\mathbf{R}^{d}} w \varphi\right|_{t=T} d x-\left.\int_{\mathbf{R}^{d}} \zeta u \varphi\right|_{t=\tau} d x
$$

for all $\varphi \in C^{2,1}\left(\mathbf{R}^{d} \times[\tau, T]\right)$ such that $\varphi(\cdot, t)$ has compact support for each $t \in[\tau, T]$.

For each cut-off function $\zeta$ and each $\tau \in(0, T),(1.9)$ has a unique weak solution $w$ which has compact support as a function of $x$ for all $t \in[\tau, T]$. To prove this assertion let $R \equiv \inf \left\{r \in \mathbf{R}^{+}: \operatorname{supp} \zeta \subset B_{r}(0)\right\}$ and set $\Gamma_{r}=B_{r}(0) \times(\tau, T]$. By Theorem 13 of $[3]^{2}$ for each $r>R$ the boundary value problem

$$
\partial v / \partial t=\Delta\left(v^{m}\right) \quad \text { in } \Gamma_{r}, \quad v=\zeta u \quad \text { on } \partial_{p} \Gamma_{r}
$$

has a unique weak solution $v_{r}(x, t)$. If $w$ is a weak solution of (1.9) with compact support, then $w \mid \Gamma_{r}=v_{r}$ for all sufficiently large $r$. Consequently, $w$ is unique. To prove existence we observe that there exists an $R^{*} \in \mathbf{R}^{+}$such that

$$
\operatorname{supp} v_{r} \subset \Gamma_{r}
$$

for all $r>R^{*}$. This follows from Proposition 1.1 by comparing $v_{r}$ with an appropriately scaled Barenblatt solution (cf. $\S 3$ below). Define

$$
w_{r}= \begin{cases}v_{r} & \text { for }|x| \leqslant r \\ 0 & \text { for }|x|>r .\end{cases}
$$

Then, in view of (1.12), there exists a function $w(x, t)$ such that $w=w_{r}$ for all $r>R^{*}$. It is easy to verify that $w$ satisfies (1.10) and is therefore a weak solution of (1.9). We will call $w$ the compact weak solution of (1.9). More generally we will call any weak solution of (1.1) in $S_{T}$ a compact weak solution if it has compact support as a function of $x$ for all $t \in(0, T)$.

Proposition 1.3 (Global Comparison Principle). Let $u$ be a continuous weak solution of (1.1) in $S_{T}$ for some $T>0$. For each fixed $C_{0}^{\infty}\left(\mathbf{R}^{d}\right)$ function $\zeta$ with values $[0,1]$ and each $\tau \in(0, T)$, let $w$ denote the weak solution of the corresponding (1.9). Then $u \geqslant w$ in $\mathbf{R}^{d} \times[\tau, T]$.

Proof. According to Proposition 1.2, $u$ is a weak solution of (1.4) in $\Gamma_{r}$ with boundary values $g=u \mid \partial_{p} \Gamma_{r}$. Since $u\left|\partial_{p} \Gamma_{r} \geqslant \zeta u\right| \partial_{p} \Gamma_{r}$, it follows from Proposition 1.1 that $u \geqslant v_{r}$ in $\bar{\Gamma}_{r}$, where $v_{r}$ denotes the solution of (1.11). However, as we observed above, $v_{r} \equiv w$ for all sufficiently large $r$. Thus $u \geqslant w$ in $\bar{\Gamma}_{r}$ for all sufficiently large $r$ and the assertion follows by letting $r \rightarrow+\infty$.

REMARK 1.4. Let $u$ be a continuous weak solution of (1.1) in $S_{T}$ for some $T>0$ and let $z$ be a compact (continuous) weak solution. If $u(\cdot, \tau) \geqslant z(\cdot, \tau)$ in $\mathbf{R}^{d}$ for some $\tau \in(0, T)$ then $u \geqslant z$ in $\mathbf{R}^{d} \times[\tau, T]$. To prove this choose $R$ so large that

\footnotetext{
${ }^{2}$ Theorem 13 is proved for $x \in \mathbf{R}$ in [3] but the proof can easily be extended to $x \in \mathbf{R}^{a}$.
} 
supp $z \subset \Gamma_{R}$. For $r>R+1$ let $v_{r}$ denote the solution of $(1.11)$, where $\zeta=\zeta(|x|)$ is a $C^{\infty}$ function such that

$$
\zeta(r)= \begin{cases}1 & \text { for } 0 \leqslant r \leqslant R \\ 0 & \text { for } r \geqslant R+1 .\end{cases}
$$

By Propositions 1.1-1.3, for all sufficiently large $r, u \geqslant w=v_{r} \geqslant z$ in $\Gamma_{r}$ and the assertion follows by letting $r \rightarrow \infty$. Note that, in particular, $u$ can also be a compact weak solution.

REMARK 1.5. The discussion of weak solutions of boundary value problems does not have to be restricted to cylindrical domains. In particular, the definition of weak solution, as well as Propositions 1.1 and 1.2, can be extended to smoothly bounded domains in $\mathbf{R}^{d} \times(0, T]$. Moreover, the conclusions of Proposition 1.3 and Remark 1.4 continue to hold in domains of the form $\Omega \times[\tau, T]$ with $\Omega \neq \mathbf{R}^{d}$ provided that the solutions involved are comparable on $\partial \Omega \times[\tau, T]$.

2. Monotonicity properties. In this section we consider compact weak solutions $u$ of the porous medium equation (1.1) which are assumed to be continuous in $\bar{S}_{T} \equiv \mathbf{R}^{d} \times[0, T]$ for some $T>0$. We show that such solutions behave almost radially in a sense made precise by the following result.

Proposition 2.1. Let $u$ be a compact weak solution of (1.1) which is continuous in $\bar{S}_{T}$ for some $T>0$. If $\operatorname{supp} u(\cdot, 0) \subset B_{1}(0)$ then

$$
\inf _{x \in B_{r}} u(x, t) \geqslant \max _{x \in \partial B_{r+2}} u(x, t)
$$

for all $r \in \mathbf{R}^{+}$and $t \in[0, T]$.

The proof of Proposition 2.1 is based on the following lemma which is a consequence of the global comparison principle. For $x \in \mathbf{R}^{d}$ we write $x=\left(y, x_{d}\right)$ where $y=\left(x_{1}, \ldots, x_{d-1}\right) \in \mathbf{R}^{d-1}$.

LEMMA 2.2. Let $u$ be a compact weak solution of (1.1) which is continuous in $\bar{S}_{T}$. If $K \equiv \operatorname{supp} u(\cdot, 0)$ is compact and $K \subset\left\{x \in \mathbf{R}^{d}: x_{d}>0\right\}$, then $u\left(y, x_{d}, t\right) \geqslant$ $u\left(y,-x_{d}, t\right)$ for all $y \in \mathbf{R}^{d-1}, x_{d} \in \mathbf{R}^{+}$, and $t \in[0, T]$.

Proof. Set $v(x, t)=u\left(y,-x_{d}, t\right)$. Then

$$
v(y, 0, t)=u(y, 0, t) \text { for all } y \in \mathbf{R}^{d-1} \text { and } t \in[0, T],
$$

and

$$
v\left(y, x_{d}, 0\right)=u\left(y,-x_{d}, 0\right)=0 \leqslant u\left(y, x_{d}, 0\right) \text { for all } y \in \mathbf{R}^{d-1} \text { and } x_{d}>0 .
$$

Since $u$ and $v$ are compact continuous weak solutions we can apply Remarks 1.4 and 1.5 to conclude that

$$
u\left(y,-x_{d}, t\right)=v\left(y, x_{d}, t\right) \leqslant u\left(y, x_{d}, t\right) \text { in } \mathbf{R}^{d-1} \times \mathbf{R}^{+} \times[0, T] .
$$

Proof of Proposition 2.1. Fix $x^{0} \in B_{r}$ and $x^{1} \in \partial B_{r+2}$. Let $\Pi$ denote the hyperplane of points in $\mathbf{R}^{d}$ which are equidistant from $x^{0}$ and $x^{1}$. Thus

$$
\Pi=\left\{x \in \mathbf{R}^{d}:\left\langle x, x^{0}-x^{1}\right\rangle=\left\langle\frac{1}{2}\left(x^{0}+x^{1}\right), x^{0}-x^{1}\right\rangle\right\},
$$


where $\langle\cdot, \cdot\rangle$ denotes the usual scalar product in $\mathbf{R}^{d}$. It is easy to verify that

$$
d(\Pi,\{0\})=\frac{1}{2} \frac{(r+2)^{2}+\left|x^{0}\right|^{2}}{\left|x^{0}-x^{1}\right|}>\frac{(r+2)^{2}}{4(r+1)} \geqslant 1 .
$$

Therefore $x^{0}$ and $\operatorname{supp} u(\cdot, 0)$ are in the same half-space with respect to $\Pi$. Moreover, $x^{1}$ is the reflection of $x^{0}$ in $\Pi$. Thus, by Lemma $2.2, u\left(x^{0}, t\right) \geqslant u\left(x^{1}, t\right)$, and the assertion follows since $x^{0} \in B_{r}$ and $x^{1} \in \partial B_{r+2}$ are arbitrary.

3. A Harnack-type estimate. In this section we prove a weak Harnack-type estimate for solutions of the porous medium equation (1.1). Specifically, we estimate the mass initially contained in a ball in terms of the radius of the ball and the density at the center of the ball at some later time. For convenience in stating the result we define $\kappa=d(m-1)+2$.

THEOREM 3.1. Let $u$ be a solution of the porous medium equation (1.1) which is continuous in $\bar{S}_{T}$ for some $T>0$. There exists a constant $C=C(d, m) \in \mathbf{R}^{+}$such that for any $\xi \in \mathbf{R}^{d}$,

$$
\int_{B_{r}(\xi)} u(x, 0) d x \leqslant C\left\{r^{\kappa /(m-1)} T^{-1 /(m-1)}+T^{d / 2} u^{\kappa / 2}(\xi, T)\right\} .
$$

For any $\alpha \in \mathbf{R}^{+}$,

$$
U(x, t)=\alpha|x|^{2 /(m-1)}\left(1-t / T^{*}\right)^{-1 /(m-1)}
$$

is a continuous solution of $(1.1)$ in $\bar{S}_{T}$ for any $T<T^{*}=(m-1) / 2 m(m+1) \alpha^{m-1}$. Let $\omega_{d}$ denote the surface area of the unit ball in $\mathbf{R}^{d}$. Then

$$
\int_{B_{r}(0)} U(x, 0) d x=\alpha \omega_{d} r^{\kappa /(m-1)}=\omega_{d}\left\{\frac{m-1}{2 m(m+1) T^{*}}\right\}^{1 /(m-1)} r^{\kappa /(m-1)},
$$

and it follows that the estimate (3.1) is sharp in this case.

Theorem 3.1 is an almost immediate consequence of the following result, which is of some independent interest.

Proposition 3.2. Let $u$ be a continuous weak solution of $(1.1)$ in $\bar{S}_{1}$ and set

$$
M \equiv \int_{B_{1}(0)} u(x, 0) d x .
$$

There exist constants $M_{0}=M_{0}(d, m) \in \mathbf{R}^{+}$and $E=E(d, m) \in \mathbf{R}^{+}$such that

$$
u(0,1) \geqslant E M^{2 / \kappa}
$$

provided that $M>M_{0}$.

In the proof of Proposition 3.2 we make extensive use of the explicit form of the Barenblatt-Pattle [5] solution of (1.1),

$$
B(x, t) \equiv t^{-d / \kappa}\left\{\left(1-F r^{2} t^{-2 / \kappa}\right)^{1 /(m-1)}\right\}^{+},
$$


where $F=(m-1) / 2 m \kappa$ and $r=|x|$. The total mass $G=G(d, m) \in \mathbf{R}^{+}$carried by $B$ is given by

$$
G \equiv \int_{\mathbf{R}^{d}} B(x, t) d x
$$

For any $\mu \in \mathbf{R}^{+}$define

$$
u_{\mu}(x, t) \equiv \mu^{-2 /(m-1)} B(\mu x, t) .
$$

It is easy to verify that $u_{\mu}$ is a solution of (1.1). Moreover,

$$
\int_{\mathbf{R}^{d}} u_{\mu}(x, t) d x=\mu^{-2 /(m-1)} \int_{\mathbf{R}^{d}} B(\mu x, t) d x=\mu^{-\kappa /(m-1)} G .
$$

Note that supp $u_{\mu}(\cdot, 1)=\bar{B}_{1 / \sqrt{F} \mu}(0)$. Thus the lower bound on $M$ in the statement of Proposition 3.2 is essential.

Proof OF Proposition 3.2. If we choose $\mu=(2 G / M)^{(m-1) / \kappa}$ then

$$
\int_{\mathbf{R}^{d}} u_{\mu}(x, t) d x=\frac{M}{2} .
$$

Moreover, if we take $t_{0}=(4 \sqrt{F} \mu)^{\kappa}$, then

$$
\operatorname{supp} u_{\mu}\left(\cdot, t_{0}\right)=\bar{B}_{4}(0) \text {. }
$$

Note that with these choices of $\mu$ and $t_{0}$,

$$
\mu^{-2 /(m-1)} t_{0}^{-d / \kappa}=(4 \sqrt{F})^{-d} M / 2 G,
$$

so that

$$
u_{\mu}\left(x, t_{0}\right) \sim M \text { for } x \in B_{3}(0) .
$$

Moreover, $M$ large implies that both $\mu$ and $t_{0}$ are small.

Assume temporarily that $u$ is a compact weak solution with

$$
\operatorname{supp} u(\cdot, 0) \subset B_{1}(0) .
$$

Recall that, by hypothesis, $u(\cdot, 0)$ is continuous.

Given $\delta>0$ we can choose $r(t)$ such that

$$
u_{\mu}\left(x, t+t_{0}\right)=\delta u_{\mu}\left(0, t+t_{0}\right)
$$

for $x \in \partial B_{r(t)}$, that is, we choose

$$
r(t)=\left(1-\delta^{m-1}\right)^{1 / 2}\left(t+t_{0}\right)^{1 / \kappa} / \mu F^{1 / 2} .
$$

In view of our choice of $\mu$ and $t_{0}$,

$$
r(0)=4\left(1-\delta^{m-1}\right)^{1 / 2} \text {. }
$$

Thus we can fix $\delta$ so that $3<r(0)<4$. Since supp $u(\cdot, 0) \subset B_{1}(0) \subset B_{3}(0)$, it follows that $u(x, t)<u_{\mu}\left(x, t+t_{0}\right)$ for $x \in \partial B_{r(t)}$ and $t>0$ sufficiently small. We now distinguish two cases.

Case 1. There exists a $t_{1} \in(0,1)$ and an $x^{1} \in \partial B_{r\left(t_{1}\right)}$ such that

$$
u\left(x^{1}, t_{1}\right)=u_{\mu}\left(x^{1}, t_{1}+t_{0}\right)=\delta u_{\mu}\left(0, t_{1}+t_{0}\right) .
$$


Let $\rho(t) \equiv r(t)-2$. By Proposition 2.1,

$$
\inf _{B_{\rho\left(t_{1}\right)}} u(\cdot, t) \geqslant \max _{\partial B_{r(t, 1)}} u(\cdot, t)
$$

so that, in particular,

$$
\inf _{B_{\rho\left(t_{1}\right)}} u(\cdot, t) \geqslant u\left(x^{1}, t_{1}\right)=\delta u_{\mu}\left(0, t_{1}+t_{0}\right) .
$$

Choose $L=L(d, m) \in \mathbf{R}^{+}$so large that

$$
\operatorname{supp} u_{\mu L}\left(\cdot, t_{1}+t_{0}\right)=L^{-1} \operatorname{supp} u_{\mu}\left(\cdot, t_{1}+t_{0}\right) \subset B_{\rho\left(t_{1}\right)}
$$

and $L^{(m-1) / 2} \geqslant \delta^{-1}$. Then

$$
\max _{B_{\rho\left(t_{1}\right)}} u_{\mu L}\left(\cdot, t_{1}+t_{0}\right)=L^{-(m-1) / 2} u_{\mu}\left(0, t_{1}+t_{0}\right) \leqslant \delta u_{\mu}\left(0, t_{1}+t_{0}\right) \leqslant \inf _{B_{\rho\left(t_{1}\right)}} u\left(\cdot, t_{1}\right)
$$

and

$$
u_{\mu L}\left(x, t_{1}+t_{0}\right)=0 \leqslant u\left(x, t_{1}\right) \text { for } x \in \mathbf{R}^{d} \backslash B_{\rho\left(t_{1}\right)} .
$$

Thus it follows from Remark 1.4 that $u(x, t) \geqslant u_{\mu L}\left(x, t+t_{0}\right)$ in $\mathbf{R}^{d} \times\left[t_{1}, 1\right]$. In particular,

$$
\begin{aligned}
u(0,1) & \geqslant u_{\mu L}\left(0,1+t_{0}\right)=(L \mu)^{-2 /(m-1)}\left(1+t_{0}\right)^{-d / \kappa} \\
& \geqslant 2^{(d-2) / \kappa} G^{-2 / \kappa} L^{-2 /(m-1)} M^{2 / \kappa} .
\end{aligned}
$$

Case 2. Suppose that $u<u_{\mu}$ on $\partial B_{r(t)}$ for all $t \in[0,1)$. Since

$$
u_{\mu}\left(x, t_{0}\right) \geqslant 0=u(x, 0) \text { for }|x| \geqslant r(0)>3,
$$

it follows from Remarks 1.4 and 1.5 that

$$
u_{\mu}\left(x, t+t_{0}\right) \geqslant u(x, t) \text { in }\{(x, t):|x| \geqslant r(t), 0 \leqslant t \leqslant 1\} .
$$

Thus, in view of (3.2) we have

$$
\begin{aligned}
M & \leqslant \int_{\mathbf{R}^{d}} u(x, 0) d x=\int_{\mathbf{R}^{d}} u\left(x, \frac{1}{2}\right) d x \\
& =\int_{B_{r(1 / 2)}} u\left(x, \frac{1}{2}\right) d x+\int_{\mathbf{R}^{d} \backslash B_{r(1 / 2)}} u\left(x, \frac{1}{2}\right) d x \leqslant \int_{B_{r(1 / 2)}} u\left(x, \frac{1}{2}\right) d x+\frac{M}{2},
\end{aligned}
$$

so that

$$
\int_{B_{r(1 / 2)}} u\left(x, \frac{1}{2}\right) d x \geqslant \frac{M}{2} .
$$

We now distinguish two subcases.

Case 2a. There exists an $x \in \mathbf{R}^{d} \backslash B_{3}$ such that $u\left(x, \frac{1}{2}\right) \geqslant \gamma M^{2 / \kappa}$, where $\gamma$ will be specified below. By Proposition 2.1,

$$
u\left(0, \frac{1}{2}\right) \geqslant \gamma M^{2 / \kappa} \text {. }
$$

It is shown in [2] that there exists a constant $H=H(d, m) \in \mathbf{R}^{+}$such that $t u_{t} \geqslant-H u$. Consequently, (3.5) implies

$$
u(0,1) \geqslant u\left(0, \frac{1}{2}\right) e^{-H \log 2} \geqslant \gamma e^{-H \log 2} M^{2 / \kappa} .
$$


Case 2b. For all $x \in \mathbf{R}^{d} \backslash B_{3}, u\left(x, \frac{1}{2}\right)<\gamma M^{2 / \kappa}$. Then in view of the choice of $\mu, t_{0}$ and $r(t)$, there exists a constant $J=J(d, m) \in \mathbf{R}^{+}$such that

$$
\int_{B_{r(1 / 2)} \backslash B_{3}} u\left(x, \frac{1}{2}\right) d x<\gamma M^{2 / \kappa} \frac{\omega_{d}}{d} r^{d}\left(\frac{1}{2}\right)=\gamma J M .
$$

In particular, take $\gamma=J / 4$. Then since $u_{\mu}>u$ in $\mathbf{R}^{d} \backslash B_{r(1 / 2)}$, it follows from (3.2) and (3.6) that

$$
\begin{aligned}
M & \leqslant \int_{\mathbf{R}^{d}} u\left(x, \frac{1}{2}\right) d x=\left\{\int_{B_{3}}+\int_{B_{r(1 / 2)} \backslash B_{3}}+\int_{\mathbf{R}^{d} \backslash B_{r(1 / 2)}}\right\} u\left(x, \frac{1}{2}\right) d x \\
& \leqslant \int_{B_{3}} u\left(x, \frac{1}{2}\right) d x+\frac{3 M}{4},
\end{aligned}
$$

so that

$$
\int_{B_{3}} u\left(x, \frac{1}{2}\right) d x \geqslant \frac{M}{4}
$$

By Hölder's inequality

$$
\int_{B_{3}} u^{m}\left(x, \frac{1}{2}\right) d x \geqslant K M^{m}
$$

for some $K=K(d, m) \in \mathbf{R}^{+}$. According to Lemma 2.3 of [6] there are constants $N=N(d, m) \in \mathbf{R}^{+}$and $P=P(d, m) \in \mathbf{R}^{+}$such that $u^{m}(0,1) \geqslant N M^{m}$ provided that $K M^{m}>P$. This will be the case if $M>M_{0} \equiv(P / K)^{1 / m}$. Finally, since $M>M_{0}$ and $\kappa>2$ we have

$$
u(0,1) \geqslant N^{1 / m} M_{0}^{1-2 / \kappa} M^{2 / \kappa} .
$$

To complete the proof of Proposition 3.2 we must remove the assumption (3.3). For $\nu \in(0,1)$ let $\psi_{\nu}(r)$ be a monotone $C^{\infty}\left(\mathbf{R}^{+}\right)$function such that

$$
\psi_{\nu}(r)= \begin{cases}1 & \text { for } 0 \leqslant r \leqslant \nu \\ 0 & \text { for } r \geqslant 1\end{cases}
$$

Let $w_{\nu}$ denote the solution of the initial value problem (1.9) on $\bar{S}_{T}$ with initial values $w_{\nu}(\cdot, 0)=\psi_{\nu} u(\cdot, 0)$. For all sufficiently large $\nu<1$,

$$
M_{\nu} \equiv \int_{B_{1}(0)} \psi_{\nu}(|x|) u(x, 0) d x>M_{0}
$$

Thus it follows from Proposition 1.3 and what we have proved under assumption (3.3) that

$$
u(0,1) \geqslant w_{\nu}(0,1) \geqslant E M_{\nu}^{2 / \kappa} .
$$

Since $E$ is independent of $\nu$ the assertion follows by letting $\nu \uparrow 1$.

Proof of Theorem 3.1. For fixed $r \in \mathbf{R}^{+}$define

$$
u^{*}(x, t)=r^{-2 /(m-1)} T^{1 /(m-1)} u(x r, t T) .
$$


It is easy to verify that $u^{*}$ is a continuous weak solution of $(1.1)$ in $\bar{S}_{1}$. In order to prove (3.1) it suffices to show that there exists a constant $C=C(d, m) \in \mathbf{R}^{+}$such that

$$
\int_{B_{1}(\xi)} u^{*}(x, 0) d x \leqslant C\left[1+\left\{u^{*}(\xi, 1)\right\}^{\kappa / 2}\right]
$$

Without loss of generality we can assume that $\xi$ is the origin. If

$$
\int_{B_{1}(0)} u^{*}(x, 0) d x \equiv M^{*}>M_{0},
$$

then, according to Proposition 3.2, there is a constant $E=E(d, m) \in \mathbf{R}^{+}$such that

$$
u^{*}(0,1) \geqslant E\left(M^{*}\right)^{2 / \kappa} \text {. }
$$

Thus

$$
\int_{B_{1}(0)} u^{*}(x, 0) d x \leqslant E^{\kappa / 2}\left\{u^{*}(0,1)\right\}^{\kappa / 2}
$$

so that (3.7) holds in this case. On the other hand, if

$$
\int_{B_{1}(0)} u^{*}(x, 0) d x \leqslant M_{0}
$$

then (3.7) holds for any $C \geqslant M_{0}$. Therefore, in particular (3.7) holds in all cases if we take $C=\max \left\{M_{0}, E^{\kappa / 2}\right\}$.

4. The main result. In this section we prove our main result: every continous weak solution of the porous medium equation in $S_{T}$ has a unique trace on $t=0$. The existence of the trace is an almost immediate consequence of Theorem 3.1. Most of this section is devoted to proving uniqueness.

THEOREM 4.1. Let $u$ be a continuous weak solution of (1.1) in $S_{T}$ for some $T>0$. There exists a unique nonegative Borel measure $\rho$ on $\mathbf{R}^{d}$ such that

$$
\lim _{t \downarrow 0} \int_{\mathbf{R}^{d}} \varphi(x) u(x, t) d x=\int_{\mathbf{R}^{d}} \varphi(x) \rho(d x)
$$

for all test functions $\varphi \in C_{0}\left(\mathbf{R}^{d}\right)$. Moreover, there exists a constant $C=C(d, m) \in \mathbf{R}^{+}$ such that

$$
\int_{B_{r}(0)} \rho(d x) \leqslant C\left\{r^{\kappa /(m-1)} T^{-1 /(m-1)}+T^{d / 2} u^{\kappa / 2}(0, T)\right\}
$$

for all $r \in \mathbf{R}^{+}$, where $\kappa=d(m-1)+2$.

Proof of Theorem 4.1. Existence. By Theorem 3.1, for any $\varepsilon \in(0, T)$,

$$
\int_{B_{n}(0)} u(x, \varepsilon) d x \leqslant C\left\{r^{\kappa /(m-1)}(T-\varepsilon)^{-1 /(m-1)}+(T-\varepsilon)^{d / 2} u^{\kappa / 2}(0, T)\right\}
$$


where $C$ is independent of $\varepsilon$. Thus there exists a sequence $\left\{\varepsilon_{k}\right\}$ such that $\varepsilon_{k} \downarrow 0$ and $u\left(\cdot, \varepsilon_{k}\right)$ converges weakly to a measure, that is,

$$
\lim _{k \rightarrow \infty} \int_{\mathbf{R}^{d}} \varphi(x) u\left(x, \varepsilon_{k}\right) d x=\int_{\mathbf{R}^{d}} \varphi(x) \rho(d x)
$$

for all $\varphi \in C_{0}\left(\mathbf{R}^{d}\right)$. The fact that $\rho$ satisfies (4.1) is an easy consequence of (4.2).

The measure $\rho$ whose existence we have just proved may, of course, depend on the sequence $\left\{\varepsilon_{k}\right\}$. The remainder of this section is devoted to proving that this is not the case. The main tool in the uniqueness proof is the following result which gives us some control on how fast material can escape from a given ball.

Proposition 4.2. Let $u$ be a weak solution of (1.1) which is continuous in $\bar{S}_{T}$ for some $T>0$. If

$$
0<\lambda<\int_{B_{1}(0)} u(x, 0) d x \leqslant \nu
$$

then for each $\varepsilon \in \mathbf{R}^{+}$there exists $\tau=\tau(d, \varepsilon, m, \nu, T) \in \mathbf{R}^{+}$such that

$$
\int_{B_{1+\varepsilon}(0)} u(x, t) d x \geqslant \lambda
$$

for all $t \in[0, \tau]$.

The proof of Proposition 4.2 is based on the following elementary lemmas.

LEMma 4.3. Let $u$ be a compact weak solution of (1.1) which is continuous in $\bar{S}_{T}$ for some $T>0$. If $\operatorname{supp} u(\cdot, 0) \subset B_{1}(0)$ and $\int_{\mathbf{R}^{d}} u(x, 0) d x \leqslant \nu$, then there exists a constant $P=P(d) \in \mathbf{R}^{+}$such that $u(x, t) \leqslant \nu / P \eta^{2 d-1}$ in $\left\{\mathbf{R}^{d} \backslash B_{1+\eta}(0)\right\} \times[0, T]$ for any $\eta \in \mathbf{R}^{+}$.

Proof. For any two points $x^{0}$ and $x^{1}$ in $\mathbf{R}^{d}$ let $\Pi\left(x^{0}, x^{1}\right)$ denote the hyperplane consisting of points which are equidistant from $x^{0}$ and $x^{1}$. According to Lemma 2.2 we have $u\left(x^{1}, t\right) \geqslant u\left(x^{0}, t\right)$ provided that $x^{1}$ and $B_{1}(0)$ lie in the same half-space with respect to $\Pi\left(x^{0}, x^{1}\right)$. Fix $x^{0} \in \mathbf{R}^{d} \backslash B_{1+\eta}(0)$ and define $S\left(x^{0}\right)$ to be the set of points $x \in \mathbf{R}^{d}$ such that

$$
\left|x^{0}-x\right|<\frac{\eta}{2} \text { and } \cos \theta \geqslant \frac{1+\eta / 4}{1+\eta},
$$

where $\theta$ denotes the angle between $x^{0}-x$ and $x^{0}$. For $x \in S\left(x^{0}\right)$,

$$
d\left(\Pi\left(x^{0}, x\right),\{0\}\right)=\left|x^{0}\right| \cos \theta-\frac{1}{2}\left|x^{0}-x\right| \geqslant(1+\eta) \frac{1+\eta / 4}{1+\eta}-\frac{\eta}{4}=1 .
$$

Thus $x \in S\left(x^{0}\right)$ implies that $x$ and $B_{1}(0)$ are in the same half-space with respect to $\Pi\left(x^{0}, x^{1}\right)$. We therefore have the estimate

$$
\nu \geqslant \int_{\mathbf{R}^{d}} u(x, 0) d x=\int_{\mathbf{R}^{d}} u(x, t) d x \geqslant \int_{S\left(x^{0}\right)} u(x, t) d x \geqslant\left|S\left(x^{0}\right)\right| u\left(x^{0}, t\right),
$$

and the assertion follows from the observation that $\left|S\left(x^{0}\right)\right| \geqslant P(d) \eta^{2 d-1}$. 
Lemma 4.4. Let $u$ satisfy the hypothesis of Lemma 4.3. For each $\varepsilon>0$ there exists a $\tau=\tau(d, \varepsilon, m, \nu, T) \in \mathbf{R}^{+}$such that $\operatorname{supp} u(\cdot, t) \subset B_{1+{ }_{\varepsilon}}(0)$ for all $t \in[0, \tau]$.

Proof. Let $u_{\mu}\left(x, t+t_{0}\right)$ denote the Barenblatt-Pattle solution of (1.1) defined in the proof of Proposition 3.2. Given $\varepsilon \in \mathbf{R}^{+}$one can verify from the formula for $u_{\mu}$ that there exist $t_{0}=t_{0}(d, \varepsilon, m, \nu, t) \in(0,1]$ and $\mu=\mu(d, \varepsilon, m, \nu, T) \in \mathbf{R}^{+}$such that

$$
\operatorname{supp} u_{\mu}\left(\cdot, t_{0}\right)=\bar{B}_{1+\varepsilon / 2}(0)
$$

and

$$
\left.\min _{t \in[0, T]} u_{\mu}\left(x, t+t_{0}\right)\right|_{|x|=1+\varepsilon / 4} \geqslant 4^{2 d-1} \nu / P \varepsilon^{2 d-1},
$$

where $P=P(d)$ is the constant defined in Lemma 4.3. By hypothesis, $u(x, 0)=0$ in $\mathbf{R}^{d} \backslash B_{1+\varepsilon / 4}(0)$. Therefore,

$$
u(x, 0)=0 \leqslant u_{\mu}\left(x, t_{0}\right) \quad \text { in } \mathbf{R}^{d} \backslash B_{1+\varepsilon / 4}(0) .
$$

Moreover, by Lemma 4.3 and the construction of $u_{\mu}$,

$$
u(x, t) \leqslant 4^{2 d-1} \nu / P \varepsilon^{2 d-1} \leqslant u_{\mu}\left(x, t+t_{0}\right) \quad \text { on } \partial B_{1+\varepsilon / 4}(0) \times[0, T] .
$$

It follows from Remarks 1.4 and 1.5 that

$$
u(x, t) \leqslant u_{\mu}\left(x, t+t_{0}\right) \quad \text { in }\left\{\mathbf{R}^{d} \backslash B_{1+\varepsilon / 4}(0)\right\} \times[0, T] .
$$

Thus the assertion holds for $\tau=\tau(d, \varepsilon, m, \nu, T)$ defined by

$$
\operatorname{supp} u_{\mu}\left(\cdot, \tau+t_{0}\right)=\bar{B}_{1+\varepsilon}(0) \text {. }
$$

Proof of Proposition 4.2. Fix $\sigma \in \mathbf{R}^{+}$such that

$$
0<\lambda<\sigma<\int_{B_{1}(0)} u(x, 0) d x \leqslant \nu .
$$

There exist numbers $\delta_{1}$ and $\delta_{2}$ such that $0<\delta_{1}<\delta_{2}<1$,

$$
\int_{B_{\delta_{1}}(0)} u(x, 0) d x=\lambda, \text { and } \int_{B_{\delta_{2}}(0)} u(x, 0) d x=\sigma
$$

Let $\zeta$ be a nonincreasing $C^{\infty}\left(\mathbf{R}^{+}\right)$function such that

$$
\zeta(r)= \begin{cases}1 & \text { for } 0 \leqslant r \leqslant \delta_{1}, \\ 0 & \text { for } r \geqslant \delta_{2},\end{cases}
$$

and let $w$ denote the solution of the initial value problem (1.9) with initial function $w(x, 0)=\zeta(|x|) u(x, 0)$. Clearly, $w(\cdot, 0) \leqslant u(\cdot, 0)$ in $\mathbf{R}^{d}, \operatorname{supp} w(\cdot, 0) \subset B_{1}(0)$ and

$$
\boldsymbol{\sigma} \geqslant \lambda^{*} \equiv \int_{\mathbf{R}^{d}} w(x, 0) d x \geqslant \int_{B_{\delta_{1}}(0)} w(x, 0) d x=\lambda .
$$


By Proposition 1.3, $w \leqslant u$ in $\bar{S}_{T}$. According to Lemma 4.4 there exists a $\tau=$ $\tau(d, \varepsilon, m, \sigma, T) \in \mathbf{R}^{+}$such that $\int_{B_{1+\varepsilon}(0)} w(x, t) d x=\lambda^{*}$ for all $t \in[0, \tau]$. Therefore

$$
\int_{B_{1+\varepsilon}} u(x, t) d x \geqslant \lambda^{*} \geqslant \lambda
$$

for all $t \in[0, \tau]$

Proof of Theorem 4.1. Uniqueness. By Theorem 3.1, for $t \in(0, T / 2)$,

$$
\int_{B_{1}(0)} u(x, t) d x \leqslant C(d, m)\left\{(2 / T)^{1 /(m-1)}+T^{d / 2} u^{\kappa / 2}(0, T)\right\} \equiv \nu .
$$

Since $u(x, t) \neq 0$ we may assume without loss of generality that $\int_{B_{1}(0)} u(x, t) d x \neq 0$. For each $s \in(0, T / 4)$ and arbitrary small $\delta \in \mathbf{R}^{+}$set

$$
\mu \equiv \int_{B_{1}(0)} u(x, s) d x-\delta .
$$

According to Proposition 4.2 there exists a $\tau=\tau(d, \varepsilon, m, \nu, T) \in \mathbf{R}^{+}$such that

$$
\int_{B_{1+f}(0)} u(x, t+s) d x \geqslant \int_{B_{1}(0)} u(x, s) d x-\delta
$$

for all $t$ which satisfy $0<t<\min (T / 4, \tau)$.

Suppose that $u$ converges weakly to a measure $\rho_{1}$ along the sequence $\left\{\varepsilon_{k}\right\}$ and to a measure $\rho_{2}$ along a sequence $\left\{\varepsilon_{k}^{\prime}\right\}$. Note that (4.1) must hold for both of these measures so that, in particular, they are regular. For fixed $t$ set $s=\varepsilon_{k}$ and take the limit of both sides of (4.3) as $k \rightarrow \infty$. Since $u$ is continuous for $t$ positive we find

$$
\int_{B_{1+\varepsilon}(0)} u(x, t) d x \geqslant \rho_{1}\left(B_{1}(0)\right)-\delta .
$$

Now set $t=\varepsilon_{k}^{\prime}$ and pass to the limit to get

$$
\rho_{2}\left(B_{1+e}(0)\right) \geqslant \rho_{1}\left(B_{1}(0)\right)-\delta .
$$

However, since $\varepsilon$ and $\delta$ are both arbitrary we conclude that $\rho_{2}\left(B_{1}(0)\right) \geqslant \rho_{1}\left(B_{1}(0)\right)$. On the other hand, if we repeat the argument first taking the limit with $s=\varepsilon_{k}^{\prime}$ followed by the limit with $t=\varepsilon_{k}$ we obtain $\rho_{1}\left(B_{1}(0)\right) \geqslant \rho_{2}\left(B_{1}(0)\right)$. Therefore

$$
\rho_{1}\left(B_{1}(0)\right)=\rho_{2}\left(B_{1}(0)\right) \text {. }
$$

By a simple scaling argument, $\rho_{1}$ and $\rho_{2}$ must agree on every ball in $\mathbf{R}^{d}$ so that $\rho_{1} \equiv \rho_{2}$.

\section{REFERENCES}

1. D. G. Aronson, Widder's inversion theorem and the initial distribution problem, SIAM J. Math. Anal. $12(1981), 639-651$

2. D. G. Aronson and Ph. Bénilan, Régularité des solutions de l'équation des milieux poreux dans $\mathbf{R}^{n}, \mathrm{C}$. R. Acad. Sci. Paris Sér. I. Math. 288 (1979), 103-105.

3. D. G. Aronson, M. G. Crandall and L. A. Peletier, Stabilization of solutions of a degenerate nonlinear diffusion problem, Nonlinear Anal. 6 (1982), 1001-1022.

4. Ph. Bénilan, M. G. Crandall and M. Pierre, Solutions of the porous medium equation in $\mathbf{R}^{n}$ under optimal conditions on initial values, Indiana Univ. Math. J. (to appear).

5. G. I. Barenblatt, On some unsteady motions of a liquid or a gas in a porous medium, Prikl. Mat. Mekh. 16 (1952), 67-78. 
6. L. A. Caffarelli and A. Friedman, Regularity of the free boundary of a gas flow in an n-dimensional porous medium, Indiana Univ. Math. J. 29 (1980), 361-39l.

7. A. S. Kalashnikov, The Cauchy problem in the class of increasing functions for equations of the nonstationary seepage type, Vestnik Moskov. Univ. Ser. I Mat. Meh. 6 (1963), 17-27.

8. M. Ughi, Initial values of non-negative solutions of filtration equation, J. Differential Equations 47 (1983), 107-132.

9. B. E. J. Dahlberg and C. E. Kenig, Non-negative solutions of the porous medium equation, preprint, University of Minnesota, 1983.

School of Mathematics, University of Minnesota, Minneapolis, Minnesota 55455 (Current address of D. G. Aronson)

Courant Institute of Mathematical Sciences, New York University, New York, New York 10012

Current address (L. A. Caffarelli): Department of Mathematics, University of Chicago, Chicago, Illinois 60637 